\title{
AN ENERGY BALANCE AND MOBILITY PREDICTION CLUSTERING ALGORITHM FOR LARGE-SCALE UAV AD HOC NETWORKS
}

\author{
Kun Fang-Le Ru ${ }^{*}$-Yunlong Yu - Xufeng Jia - Shuguang Liu
}

Aeronautics and Astronautics Engineering College, Air Force Engineering University, Xi'an, Shanxi 710038, China

\begin{tabular}{l} 
ARTICLE INFO \\
\hline Article history: \\
Received: 22.05 .2016$. \\
Received in revised form: 11.12.2016. \\
Accepted: 11.01 .2017$. \\
\hline Keywords: \\
Energy balance \\
Mobility prediction \\
Rf stealth of communication \\
Doppler shift \\
Larger-scale UAV ad hoc networks \\
\hline DOI: htp:/ldoi.org/10.30765/er.39.1.1
\end{tabular}

DOI: http://doi.org/10.30765/er.39.1.1

\section{Introduction}

With the development of science and technology, UAVs have become more and more popular in various fields such as military and civilian. The way to carry out tasks is from single UAV to a swarm of UAVs [1]. Aeronautical Ad Hoc Networks (AANET) is the typical development and

\footnotetext{
* Corresponding author. Tel.: +8617791658816

E-mail address: destinywe2015@sina.com
}

\begin{abstract}
:
Creating a clustering structure is considered the performance of radio frequency (RF) stealth for data link in the battlefield environment and the dynamic topology characteristic for larger-scale unmanned aerial vehicle (UAV) ad hoc networks. This problem is of a great importance-to get low intercept probability of the data link and low randomness of clustering structure. An energy balance and mobility prediction (EBMP) clustering algorithm is proposed. In the initial clustering stage, the power management for information transmission is conducted in the network layer and the MAC layer. The Doppler shift is implemented to estimate the relative speeds stability degree between neighboring UAVs when they exchange Hello packets. It can be selected as cluster head $(\mathrm{CH})$ where one UAV associates lower energy consumption with higher relative stability. In the cluster maintaining stage, a $\mathrm{CH}$ rotation process for the dynamic topology to improve resource utilization efficiency. The inter-cluster communication is enhanced by dynamic packet forwarding gateway. The simulations and analysis show that this scheme can provide better results for larger-scale UAV ad hoc networks compared to $M P B C$ and MPCR in terms of improving $\mathrm{CH}$ lifetime and throughput, reducing average delay.
\end{abstract}

application of Ad Hoc Networks. However, when the speed of UAVs is very fast, the topology changes quickly, which results in poor link quality between UAVs. The increasing control overheads threaten the throughput of the network. Meanwhile, the data link generally uses maximum peak power to transmit information [2]. However, for largescale UAV ad hoc networks, not only reliable transmission of data should be ensured, but also the 
intercept probability of communication in the complex battlefield environment should be reduced. The transmission power is set to minimum value that provides acceptable performance in order to achieve RF stealth. Energy balance is favorable for power management, which can accordingly achieve RF stealth in information transmission process [3]. Clustering is an effective method for improving the performance of large-scale UAV ad hoc networks, which can solve the problem of network expansion and satisfy the requirements of communication.

In previous work, the advantages of the clustering algorithm in Wireless Sensor Networks (WSN) have been widely studied. As research continues, the clustering algorithm for mobile nodes over hierarchical structure was investigated and compared with plane structure. Now, there has been much interest in studying the communication performance of large-scale and highly dynamic ad hoc networks by using clustering algorithm. In [4], a clustering algorithm based on probability degree is proposed by using Markov process for solving the problem of instability in communication. The node of the highest link connectivity probability is selected as the $\mathrm{CH}$. The algorithm can decrease the clustering maintenance cost. In [5], a mobility metric-based clustering (MOBIC) algorithm is proposed. The algorithm uses the power of two successively received packets to evaluate the relative motion between two mobile nodes, but the nodes need to move with similar speed and direction, which is not suitable for the UAV moves randomly. The clustering algorithm proposed in [6] called adaptive distributed weighted clustering algorithm (ADWCA). The algorithm considers mobility of the node, the average distance between neighbors, degree of nodes, and energy consumption. Its comprehensive performance is superior, but ADWCA is not especially appropriate for a highly dynamic network. In [7], a mobility prediction-based clustering (MPBC) scheme is proposed. The Doppler shift is used to estimate the neighboring nodes' relative speeds. The nodes having the smallest relative mobility in their neighborhoods are selected as the $\mathrm{CHs}$, but the speed calculation is not precise. In [8], a mobility prediction clustering routing (MPCR) algorithm is proposed. The algorithm selects the node that has the largest connectivity as the $\mathrm{CH}$ and introduces ferry nodes forward messages between different clusters. But MPCR is too dependent on ferry nodes for inter-cluster communication.

In this paper, inspired by the above work and shortcomings, we propose an energy balance and mobility prediction (EBMP) clustering algorithm. The proposed EBMP clustering algorithm includes three stages. In the initial clustering stage, we combine the transmission power management with the RF stealth of communication, which is to realize the goal of energy balance. We adopt the Doppler shift to estimate the neighboring UAVs' relative stability degree. Then we use the estimated energy consumption and relative stability degree to select the appropriate $\mathrm{UAV}$ as the $\mathrm{CH}$ and establish clusters. In the cluster maintaining stage, the $\mathrm{CH}$ rotation strategy is used to keep a stable clustering relationship for a long term. In the communication stage, we design routing algorithm of EBMP to deal with the problems of intra-cluster communication and inter-cluster communication.

\section{Cluster structure}

Without loss of generality, this paper adopts reference point group mobility (RPGM) model. The $\mathrm{CH}$ UAV acts as the reference point and it moves with random directions and speeds chosen from $[0,2 \pi]$ and $\left[v_{\min }, v_{\max }\right]$, where $v_{\min }$ and $v_{\max }$ are minimum and maximum speed of UAVs. Other CM UAVs need an additional $[0,2 \pi]$ random offset on the basis of the speed and direction of CH UAV. The movement characteristics of the CM UAVs are generally consistent with the $\mathrm{CH} \mathrm{UAV}$ in the service area. Each UAV changes its initial speed and direction only when the CM UAV is ready to leave the original cluster to join another cluster or become an orphan node.

Each UAV broadcasts Hello packets with a constant period $T$. Based on the received Hello packet, each node can establish and update a neighbor list. If $\mathrm{UAV} j$ is currently on the neighbor's list of UAV $i$, but UAV $i$ does not receive Hello packets from $\mathrm{UAV} j$ more than $a T(a>1)$, then $\mathrm{UAV} j$ is removed from the neighbor list of UAV $i$. Physical channel fading and transmission collision would affect that UAV $i$ normally receives Hello packets. Appropriate increase in the value of $a$ in the allowed range can provide a more stable cluster structure.

The backbone network should be established between clusters for inter-cluster communication 
and building of the one-hop communication subnet within the cluster. The routing information of intracluster and inter-cluster is maintained by the $\mathrm{CH}$. It has two-hop communications in the cluster at most, which use $\mathrm{CH}$ relay. The hierarchical network of the cluster structure is shown in the Fig. 1.

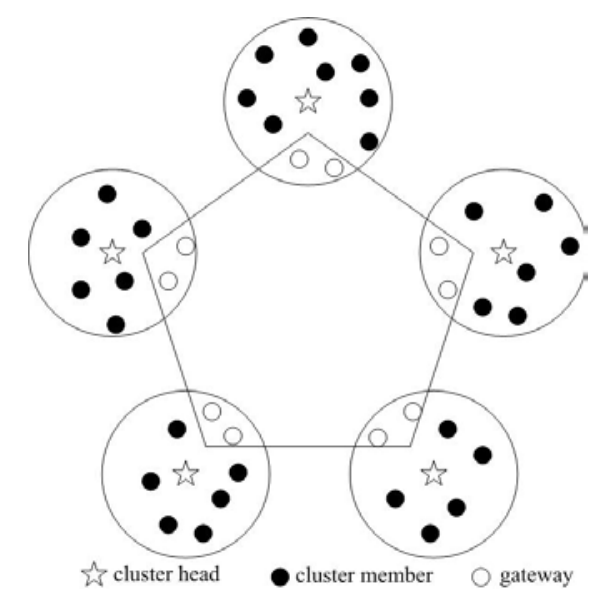

Figure 1. The hierarchical network of the cluster structure.

\section{CH selection in the EBMP algorithm}

\subsection{Energy consumption model}

According to the protocol stack system model, the information transmission types of UAVs are roughly divided into three categories: network layer packet (Hello, RREQ, RREP), MAC layer control frame (RTS, CTS, ACK), and data packet. Economical power management can reduce the intercept probability of communication and achieve RF stealth for data transmission. One UAV adjusts the transmission range in the network layer This concern whether or not the UAV has the ability to discover the neighbor UAVs. The MAC layer mainly controls the minimum area required for information transmission, which concerns space multiplexing of the entire network. Therefore, power management must compromise the pros and cons of the network layer and MAC layer to achieve a relatively optimal result.

The number and duration of data packet are more than control packet. It would lead to a waste of energy, if data packet and control packet use the same transmission power. Frequently switched transmission power will result in additional energy consumption and power switching delay in the hardware system. Therefore, it is not appropriate to change the transmission power of network layer packet and MAC layer control frame from time to time. In order to preferably achieve RF stealth of communication, the transmission power is switched by the distance between two UAVs when transmitting the data packet. The network layer packet and MAC layer control frame the use of transmission power constantly. The transmission power consists of three types of information, and it is defined as follows:

1) In order to realize the return routability in the whole network, the transmission power of RREQ must be large enough to reach information from any UAV in the network. We stipulate its transmission power as the maximum value of $P_{i j}$, where $P_{i j}$ is the transmission power between UAV $i$ and UAV $j$ that they can communicate with each other.

$$
P_{\mathrm{RREQ}}=\max \left\{P_{\mathrm{t}}\left(d_{i j}\right) \mid i, j \in N\right\}
$$

Where, $d_{i j}$ is the distance between UAV $i$ and UAV $j$, its value can be calculated from received signal strength indicator (RSSI) [9]:

$$
d_{i j}=10^{\left[27.55+\operatorname{RSSI}_{i j}(\mathrm{~dB})-20 \lg (f)+\left(\sum G_{i j}-\sum L_{i j}\right)\right] / 20}
$$

Where, $G_{i j}$ is the sum of the transmitting and receiving antenna gain of $\mathrm{UAV} i$ and $\mathrm{UAV} j, f$ is the natural frequency of the transmitter. These two are hardware system parameters that can be found in technical manuals. $L_{i j}$ is the loss of signal obtained from basic rules. RSSI can be obtained at the receiver.

When RREQ completes the route discovery, it uses the same transmission power for RREP and Hello as long as two UAVs can be received successfully.

$$
P_{\mathrm{RREP}}=P_{\text {Hello }}=P_{\mathrm{t}}\left(d_{i j}\right) \quad, \quad(i, j \in N)
$$

2) In order to achieve data transmission efficiently, we select the maximum mean value from the distance and the distance of two UAVs to determine the transmission power of MAC layer control frame.

Each CM UAV with a uniform distribution, the probability density function of the distance between any two UAVs can be expressed as follows:

$$
f(d)=2 d / R_{\max }^{2}
$$


Where, $d$ is the distance variable of any two UAVs, $R_{\max }$ is the maximum transmission range of the UAV. The mean distance value $\mathrm{E}(d)$ can be obtained by integration.

$$
\mathrm{E}(d)=\int_{0}^{R_{\max }} f(x) x d x=\frac{2}{3} R_{\max }
$$

Based on the above analysis, the transmission power of MAC layer control frame can be expressed as follows:

$$
P_{\mathrm{MAC}}=\max \left\{P_{\mathrm{t}}\left(d_{i j}\right), P_{\mathrm{t}}\left(\frac{2}{3} \mathrm{R}_{\text {max }}\right) \mid i, j \in N\right\}
$$

3) Data packet is the major part of all the UAVs that communicate in the network. Based on the wireless channel model, the transmission power is adjusted by the distance between two UAVs:

$$
\begin{gathered}
P_{\mathrm{t}}\left(d_{i j}\right)=\mathrm{b} P_{\mathrm{t}}^{\mathrm{min}}\left(d_{i j}\right)=b K P_{\mathrm{r}}^{\mathrm{min}} d_{i j} \\
K=\frac{(4 \pi)^{2}}{G_{\mathrm{t}} G_{\mathrm{r}} \lambda^{2}}
\end{gathered}
$$

Where, $P_{\mathrm{r}}^{\min }$ is a sensitivity of receiver, $G_{\mathrm{t}}$ and $G_{\mathrm{r}}$ are the transmitting and receiving antenna gain, $\lambda$ is the wavelength. It is bound to produce the loss in the process of information transmission. A coefficient $\mathrm{b}$ greater than one is multiplied before the theoretical minimum transmission power. This is to ensure that the received signal is no less sensitive from a receiver in a real case.

At transmitter, energy consumption is mainly generated by the transmitting circuit and power amplifier. At the receiver, energy consumption is done only by the receiving circuit. Suppose there are $l$ bits information to be transmitted from the $\mathrm{UAV} i$ to $\operatorname{UAV} j . E_{\mathrm{t}}^{i j}(l)$ represents the energy consumption that UAV $i$ transmit $l$ bits information to $\mathrm{UAV} j, E_{\mathrm{r}}^{i j}(l)$ represents the energy consumption that UAV $j$ receive $l$ bits information from UAV $i$, $E T_{\text {elec }}$ represents a unit bit stream energy consumption of the transmitting circuit, $E R_{\text {elec }}$ represents a unit bit stream energy consumption of the receiving circuit. $P_{\mathrm{t}}^{i j}$ represents the transmission power and determined by the types of information transmission, $r$ represents the information transmission rate. The energy consumption of transmitting and receiving $l$ bits information can be expressed as follows:

$$
\begin{gathered}
E_{\mathrm{t}}^{i j}(l)=\left(E T_{\text {elec }}+\frac{P_{t}^{i j}}{\sigma_{i}}\right) \frac{l}{r} \\
E_{\mathrm{r}}^{i j}(l)=E R_{\text {elec }} \frac{l}{r}
\end{gathered}
$$

Where, $\sigma_{i}$ is the amplifier power amplifier efficiency of $\mathrm{UAV} i, \sigma_{i} \in(0,1]$.

\subsection{Mobility prediction model}

Any UAVs are flying at a certain speed, in order to estimate the relative speed between the two UAVs and reduce the calculation complexity. Without loss of generality, we set up a coordinate system of relative motion and estimate the relative speed in approaching scenario. We see UAV $i$ as static at coordinate origin $\mathrm{O}$ and UAV $j$ as mobile with a relative speed $v_{\mathrm{r}}$ toward negative direction of the $\mathrm{x}$ axis at P. In fact, UAV $i$ and $\mathrm{UAV} j$ are both mobile. The relative speed can be estimated after UAV $i$ receives two Hello packets from UAV $j$. UAV $j$ sends a Hello packet at $\mathrm{P}$ and Q, respectively. UAV $i$ successfully receives the packets. $T$ is the time interval between two Hello packets. Fig. 2 illustrates relative mobility of two UAVs.

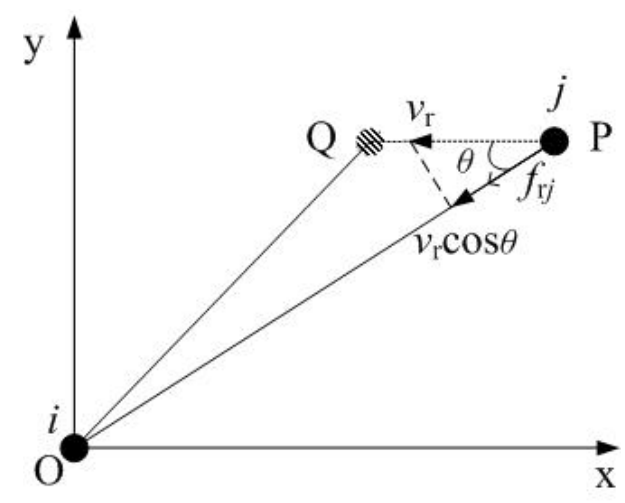

Figure 2. Relative mobility of two UAVs.

In the Fig. 2, let $f_{\mathrm{rj}}$ be the transmission frequency of Hello packets from UAV $j$ to UAV $i$ at P. $\theta$ is the included angle between $f_{\mathrm{rj}}$ and $v_{\mathrm{r}}$. According to the special theory of relativity, $f_{\text {rj }}$ satisfies the following formula with the previously mentioned transmitter natural frequency $f$ : 


$$
f_{\mathrm{rj}}=f \sqrt{1-\left(\frac{V_{\mathrm{r}}}{c}\right)^{2}}=f \sqrt{1-\beta^{2}}
$$

Where, $c$ is the speed of light.

$f_{0}$ is the receiving frequency of Hello packets at $\mathrm{UAV} i$ and can be expressed as follows:

$$
f_{0}=\frac{\sqrt{1-\beta^{2}}}{1-\beta \cos \theta} f
$$

Where $T$ is very short and $\theta$ is small, therefore, $\cos \theta$ can be approximated as follows:

$$
\cos \theta \approx \frac{d_{\mathrm{OP}}-d_{\mathrm{OQ}}}{d_{\mathrm{PQ}}}=\frac{d_{\mathrm{OP}}-d_{\mathrm{OQ}}}{v_{\mathrm{r}} T}
$$

Where, $d_{\mathrm{OP}}, d_{\mathrm{OQ}}$ can be calculated from the formula (2).

Substituting (13) in (12), we have

$$
\begin{gathered}
\left(\frac{v_{\mathrm{r}}}{c}\right)^{2}=1-A^{2} B^{2} \\
A=f_{0} / f \\
B=1-\frac{d_{\mathrm{OP}}-d_{\mathrm{OQ}}}{T c}
\end{gathered}
$$

From (14), (15) and (16), $v_{\mathrm{r}}$ can be solved as

$$
v_{\mathrm{r} i j}^{\mathrm{n}}=c \sqrt{1-\left(\frac{f_{0}}{f}\right)^{2}\left(1-\frac{d_{\mathrm{OP}}-d_{\mathrm{OQ}}}{T c}\right)^{2}}
$$

The relative speed in the receding scenario can be estimated in a similar way.

$$
v_{\mathrm{rij}}^{\mathrm{f}}=c \sqrt{1-\left(\frac{f_{0}}{f}\right)^{2}\left(1-\frac{d_{\mathrm{OP}}-d_{\mathrm{OQ}}}{T c}\right)^{2}}
$$

The only difference between (17) and (18) is $A$ and $B$ which have been defined in (15) and (16), in (17) $A>1, \quad B<1$, but in (18) $A<1, \quad B>1$.

The stability degree of $X$ can be calculated by the variance $D(X)$ [10]. Therefore, the relative stability degree of UAV $i$ can be expressed as follows:

$$
S D_{i}=\left(\sum_{j} \frac{v_{\mathrm{rij}}^{2}}{N}\right)-\left(\sum_{j} \frac{v_{\mathrm{rij}}}{N}\right)^{2}
$$

\subsection{The $\mathrm{CH}$ selection}

In order to get low intercept probability of the data link and low randomness of clustering structure, we calculate the energy consumption and the relative stability degree of each UAV and formulate the weights to select $\mathrm{CH} \mathrm{UAV}$.

Setting a period of time $\tau(\tau<\mathrm{a} T)$, which can ensure that any UAV has sufficient time to receive the information from neighbor UAVs within $\tau$. The $\mathrm{CH}$ selection adopts the Analytic Hierarchy Process (AHP) to determine the weight of each index. UAV $i$ become the $\mathrm{CH}$ which has the largest Weight.

$$
\text { Weight }_{i}=0.26 e^{-E_{i}}+0.74 e^{-S D_{i}}
$$

\section{Clusters formation}

In order to ensure the stability of the whole network, it can reduce the likelihood of congestion that set the bandwidth of intra-cluster and intercluster to be $B_{1}$ and $B_{2}$. If the whole network can be divided into $m$ clusters, we have $B_{2}=m B_{1}$. We can get the optimal number of UAVs in a cluster according to the conclusion about [11]:

$$
n=N / m=N^{\frac{3}{4}} \sqrt{\frac{B_{1}}{B_{2}}}
$$

The clusters formation can be described as follows: 1) UAV $i$ calculated its Weight $t_{i}$ from other UAVs in its neighbor list. The Weight $t_{i}$ is updated based on the latest neighbor list. Upon receiving the Weight from its neighbors, UAV $i$ compares them with its own Weight . If its Weight $_{i}$ is the most, UAV $i$ becomes a $\mathrm{CH}$ and broadcasts the HEAD announcement to its neighbors. The HEAD announcement contains the address of UAV $i$ and its own Weight $t_{i}$. Otherwise, UAV $i$ waits for the HEAD announcement from other UAVs. Each UAV independently operates the $\mathrm{CH}$ selection algorithm of EBMP.

2) CH UAV $i$ initializes a $N^{*} 2$ matrix $\mathbf{G}_{\mathbf{J}}$, each line is used to store a JOIN announcement other UAVs. The JOIN announcement includes the address of the applicant UAVs and the amount of HEAD announcement in their own $\mathbf{G}_{\mathrm{H}}$. The other UAVs initialize $N^{*} 2$ matrix $\mathbf{G}_{\mathrm{H}}$. When other UAVs receive 
the HEAD announcement, they will save the HEAD announcement in their own $\mathbf{G}_{\mathrm{H}}$.

3) Waiting for a period of time $\tau$. UAV $j$ will send the JOIN announcement to apply to join the cluster if there is only one HEAD announcement in its $\mathbf{G}_{\mathrm{H}}$. UAV $j$ will select the largest Weight of UAVs and send the JOIN announcement if there are many HEAD announcements in its $\mathbf{G}_{\mathrm{H}}$.

4) CH UAV $i$ counts the rows $n_{\mathrm{J}}$ of its $\mathbf{G}_{\mathrm{J}}$ after a period of time $\tau$. It will send AGREE announcements to all the UAVs which are in the $\mathbf{G}_{\mathbf{J}}$ if $n_{\mathrm{J}}<n$. If $n_{\mathrm{J}}>n$, CH UAV $i$ preferentially send the AGREE announcements to UAVs whose second column value of $\mathbf{G}_{\mathrm{J}}$ is 1 (UAV $j$ only received one HEAD announcement). The aim is to reduce the state of orphan UAV. Then CH UAV $i$ selects the smaller row index value ( $\mathrm{CH}$ UAV $i$ early received the JOIN announcements, means shorter distance between two UAVs) of UAVs in the $\mathbf{G}_{\mathrm{J}}$ and send AGREE announcements to them, just like the first in first out (FIFO) of the stack structure. CH UAV $i$ specifies $n$ UAVs in the cluster at most, make $n_{\mathrm{J}}=n$. AGREE announcement includes the address of $\mathrm{CH}$ UAV $i$ and IP address that assigned for each CM UAVs.

5) UAV $j$ removes $\mathrm{CH}$ UAV $i$ from its $\mathbf{G}_{\mathrm{H}}$ if it does not receive the AGREE announcement after a period of time $\tau$. If its $\mathbf{G}_{\mathrm{H}}$ is void, UAV $j$ becomes orphan UAV. If its $\mathbf{G}_{\mathrm{H}}$ is non-void, UAV $j$ selects the largest Weight of UAVs in the modified $\mathbf{G}_{\mathrm{H}}$ and send the JOIN announcement to it. When UAV $j$ successfully becomes a CM UAV, it will clear its $\mathbf{G}_{\mathrm{H}}$ except the address of CH UAV, which is used to identify the cluster that UAV $j$ belongs to.

\section{The $\mathrm{CH}$ rotations}

Energy consumption of $\mathrm{CH}$ UAV is much larger than CM UAVs. Since CH UAV not only maintains the topology within the cluster, but also updates the routing list at any moment. The relative mobility between a $\mathrm{CH}$ UAV and all its CM UAVs may change with time. To keep a stable clustering relationship for a long term, it may be necessary to have a different UAV service as $\mathrm{CH}$ UAV under some conditions.

In EBMP clustering algorithm, CH UAV updates its neighbor list with CM UAVs based on the received Hello packets. It will implement $\mathrm{CH}$ rotations if the following two conditions occurr:
1) If CM UAVs does not receive Hello packet from CH UAV $i$ after a long time aT, CH UAV $i$ is considered that have lost contact or been shot down. At this time, CM UAVs remove $\mathrm{CH}$ UAV from their $\mathbf{G}_{\mathrm{H}}$ and re-select a new $\mathrm{CH} \mathrm{UAV}$.

2) When CH UAV $i$ find that its Weight $_{i}$ is below more than half of CM UAVs, CH UAV $i$ broadcasts a ROTATION announcement to CM UAVs. CM UAVs begin the $\mathrm{CH}$ selection algorithm when receive the ROTATION announcement. The previous $\mathrm{CH}$ UAV $i$ does not participate in the competition and no longer serves as the $\mathrm{CH}$ and only waits to receive the HEAD announcement.

The new $\mathrm{CH}$ UAV modifies the previous $\mathrm{CH}$ UAV and its own IP address base on the IP address of originally assigned for the cluster, which can avoid introducing unnecessary cost after $\mathrm{CH}$ rotations and improve the efficiency of the cluster.

\section{Routing algorithm of the EBMP}

\subsection{Intra-cluster communication}

CH UAV directly sends the data to CM UAVs to lookup its neighbor list. When CM UAV $j$ wants to send the data, it first lookups whether the destination UAV in its neighbor list. If the destination UAV is in the neighbor list, then it communicates directly to the destination UAV. Otherwise, UAV $j$ sends data to $\mathrm{CH} \mathrm{UAV}, \mathrm{CH}$ UAV acts as a relay.

\subsection{The inter-cluster communication}

Priority of routing will be given to those who have the least energy consumption (the shorter distance) by $\mathrm{CH}$ UAV when broadcasts RREQ. Since CM UAVs are within one hop range for their $\mathrm{CH} U A V$. If one CH UAV finds that destination UAV is in its neighbor list when received RREQ, it will immediately reply RREP to source $\mathrm{CH}$. Therefore, the communication routing is established which can provide a viable path for information transmission between different clusters of lower energy consumption.

However, the communication between the different clusters must occur through the gateway. It is mainly due to different clusters use the different spreading codes. The gateway can choose to connect two different clusters. A UAV cannot 
always serve as a gateway due to the random movement. For this reason, we modified RREP packet by adding two fields for storing the sequence number of the gateway and assigned IP address. The modified RREP packet as shown in Fig. 3.

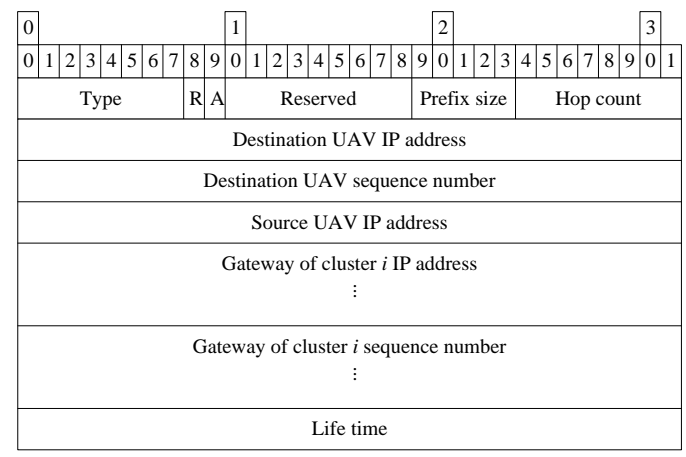

Figure 3. The modified RREP packet.

The $\mathrm{CH}$ of the source UAV assigns the gateway and IP addresses when received RREP. The source UAV only submits the data to $\mathrm{CH}$ UAV or the assigned gateway. $\mathrm{CH} \mathrm{UAV}$ and the assigned gateway complete the information transmission through the established routing.

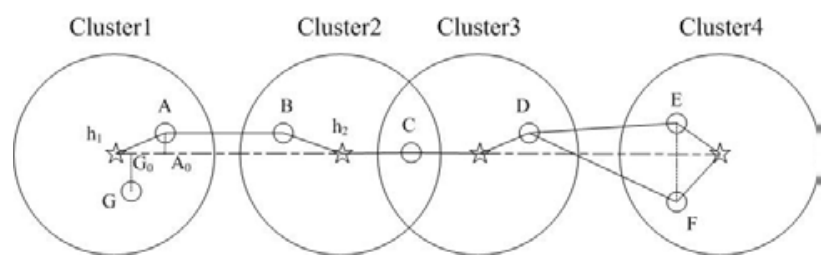

Figure 4. The gateway selection.

The selection of gateway uses the method of the shortest projection distance. One UAV is selected as gateway that it has the shortest distance between its projection point and the adjacent $\mathrm{CH}$ UAV. The projection point is on the line connecting two $\mathrm{CH}$ UAVs. For example, in the cluster 1 of the Fig. 4, the projection points on the line connecting the $\mathrm{CH}$ UAV $h_{1}$ and CH UAV $h_{2}$ of UAV A and UAV G are $A_{0}$ and $G_{0}$. The UAV A selects the gateway because of $\mathrm{A}_{0} \mathrm{~h}_{2}<\mathrm{G}_{0} \mathrm{~h}_{2}$.

But the projection points may coincide with one point, which corresponds to two or more UAVs that have the shortest distance with the adjacent $\mathrm{CH}$ UAV. One UAV is selected as the gateway and the other is the standby gateway. Which UAV $\mathrm{E}$ or $\mathrm{F}$ can be selected as gateway in the cluster 4 of the
Fig. 4. We use the literature [12] proposed the concept of entropy:

$$
K=\frac{1}{1+e^{-\left\{0.5\left[\mathrm{H}_{1}(X)-\mathrm{H}_{2}(X)\right]-5\right\}}}=\frac{1}{1+e^{-(0.5 \Delta \mathrm{H}-5)}}
$$

Where, $K \in(0,1] . \mathrm{H}_{1}(X), \mathrm{H}_{2}(X)$ denotes the entropy of UAV $X$ at time $t_{1}, t_{2} . \Delta H$ is the difference of the entropy in the $t_{1}-t_{2}$. The larger of the value $K$ is, the greater the data fluctuates, and vice versa. Therefore, one UAV that has the largest value of $K$ will be selected as the gateway. The UAV that has the smallest $r$ value $K$ is the standby gateway.

\section{Simulation and analysis}

The simulation software is NS-2.29 and MATLAB. There are 200 UAVs distributing in $500 \mathrm{~km} * 500 \mathrm{~km}$ service area. Dynamic TDMA is adopted in the cluster. The competition slots have the same number of UAVs. The number of data slots is 1.5 times than the competition slots. When two UAVs are within each other's communication distance, the packet loss probability that transmitted Hello packet is 0.1 .

Table 1. Parameter settings

\begin{tabular}{|c|c|}
\hline Parameters & Value \\
\hline MAC protocol & IEEE802.11 \\
\hline number of UAVs & 200 \\
\hline speed of UAVs & $70 \mathrm{~m} / \mathrm{s} \sim 150 \mathrm{~m} / \mathrm{s}$ \\
\hline data packet length & $5120 \mathrm{bits}$ \\
\hline channel rate & $4 \mathrm{Mb} / \mathrm{s}$ \\
\hline bandwidth & $30 \mathrm{Mb} / \mathrm{s}$ \\
\hline carrier frequency & $2.4 \mathrm{GHz}$ \\
\hline data packets delay & $0.1 \mathrm{~s}$ \\
\hline
\end{tabular}

For comparison, MPCR and MPBC clustering algorithm are indicated with the EBMP. In order to make each clustering algorithm in the same simulation condition, in the simulation, the ferry UAV is canceled from MPCR algorithm. The average $\mathrm{CH}$ lifetime, throughput, and average delay are compared in order to analyze the performance of three clustering algorithms. 


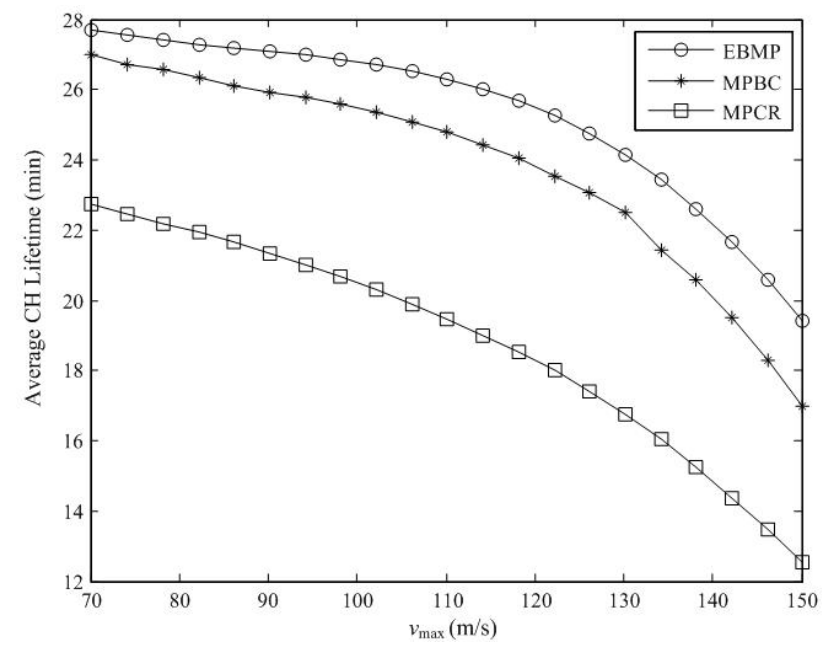

Figure 5. The average $\mathrm{CH}$ lifetime versus $v_{\max }$.

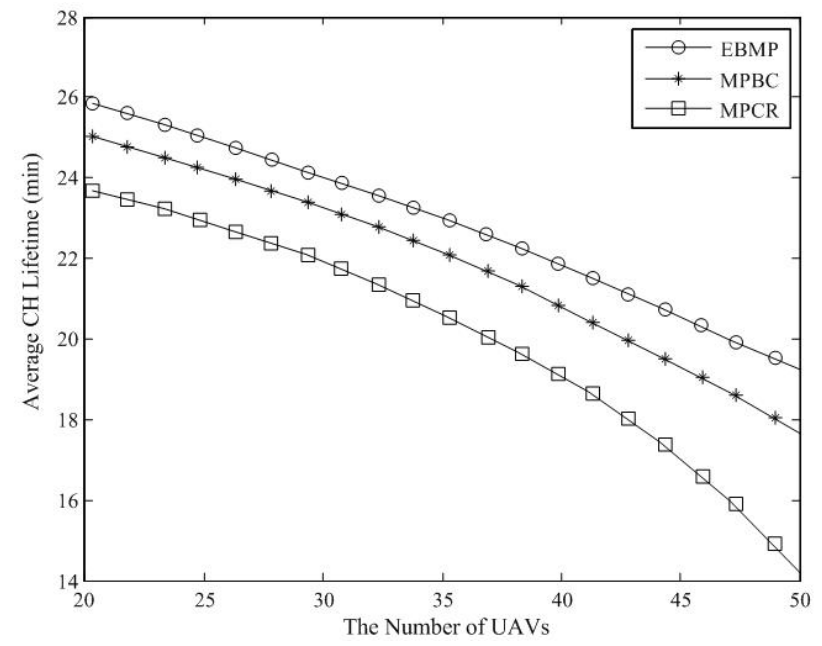

Figure 6. The average $\mathrm{CH}$ lifetime versus the number of UAVs.

In Figure 5, there are 30 UAVs in one cluster. The average $\mathrm{CH}$ lifetime reduces rapidly when the $v_{\max }$ increases. The larger the $v_{\max }$, the smaller is stability degree. Meanwhile, the delivery rate of packets will reduce, and extra energy would be consumed to retransmit the packets. It can be seen that the EBMP has larger average of $\mathrm{CH}$ lifetime than MPBC and MPCR. The MPCR clustering algorithm requires a lot of information to update the cluster topology structure. $\mathrm{O}$ the one hand, the EBMP considers the energy consumption of information transmission relieving the pressure of $\mathrm{CH} \mathrm{UAV}$ and the network overhead. On the other hand, using the minimum transmission power to send information and ensure the information can be received exactly, which can decrease the intercept probability of the data link.
In Fig. 6, the average $\mathrm{CH}$ lifetime decreases gradually with the number of UAVs increasing in one cluster at $v_{\max }=90 \mathrm{~m} / \mathrm{s}$. The variance of relative speed will be violent due to many UAVs in the cluster, which makes the Weight of CH UAV early below more than half of CM UAVs. Three clustering algorithms have the same tendency before 35 UAVs in one cluster. When the number of UAVs exceeds 35, for MPCR, its delivery rate of packets increases, but $\mathrm{CH}$ UAV requires a lot of information to maintain its topology, which makes the average $\mathrm{CH}$ lifetime to rapidly decline. That sacrifices the control overhead and only slightly improves the delivery rate of packets. It is substantially the same tendency with the EBMP and MPBC. Two clustering algorithms use the Doppler shift to predict relative speed between two UAVs. The EBMP uses variance to calculate the stability degree differing from the sliding average of MPBC. The EBMP adopts power control to reduce intercept probability of the data link. The limitations of $\mathrm{CH}$ rotations are more detailed.

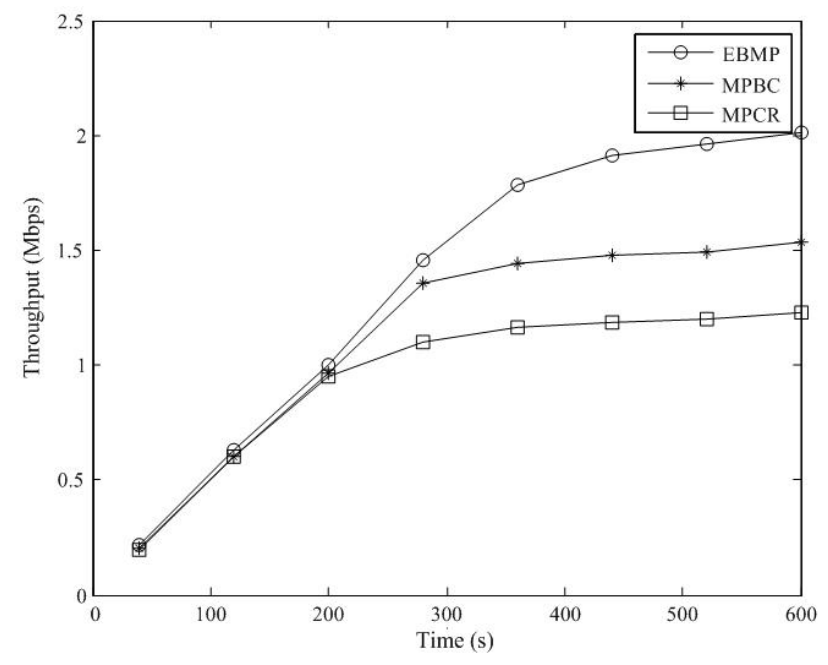

Figure 7. Throughput versus time.

Figure 7 depicts the throughput versus time at 30 UAVs in one cluster and $v_{\max }=90 \mathrm{~m} / \mathrm{s}$. Before 200s, three clustering algorithms have little difference. After 200s, there are many differences for three algorithms. Because MPCR canceled ferry UAVs in the simulation, it has little effect on intra-cluster communication, but the inter-cluster communication faces being capped. The inter-cluster communication of MPBC uses two CH UAVs from different adjacent clusters. It needs the step of decoding due to UAVs in different cluster and use 
different spreading codes, which affects the throughput of the whole network. The EBMP adds the gateway, which is not fixed one UAV. On the one hand, it can adapt to the dynamic topology of the network. On the other hand, the gateway relieves the pressure of $\mathrm{CH} \mathrm{UAV}$ and coordinates the different spreading codes between the adjacent clusters, which increase the throughput.

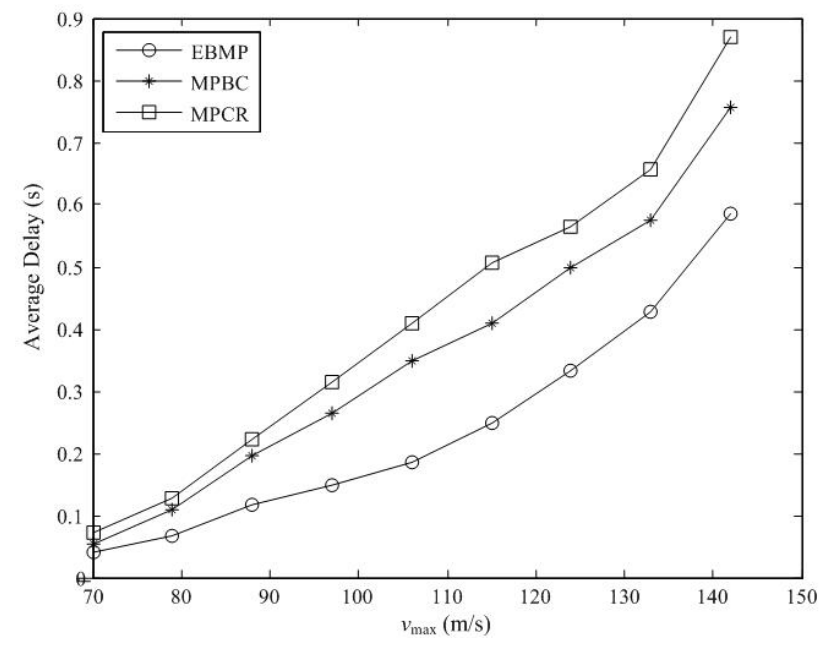

Figure 8. Average delay versus $v_{\max }$.

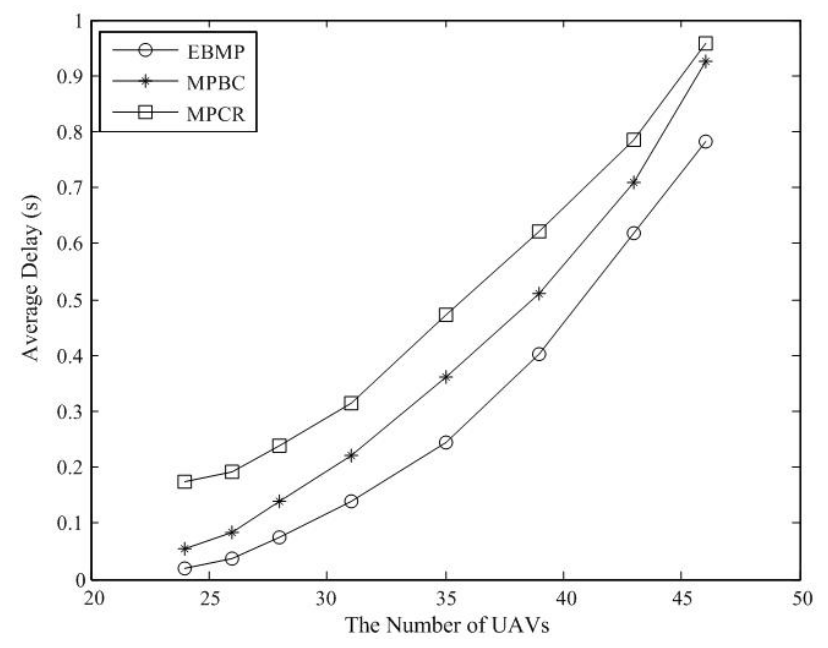

Figure 9. Average delay versus the number of UAVs.

In Fig. 8, there are 30 UAVs in one cluster. The average delay rises rapidly when the $v_{\max }$ increases. The larger the $v_{\max }$, the faster the outage probability of the link increases. MPCR canceled ferry UAVs, and the data cannot be efficiently forwarded when the queue is full. MPBC pays more attention to the link lifetime, which is bound to decline when the speed of UAV increases. The data loss and retransmissions make the average delay increase rapidly. In the EBMP, CH UAV flexibly chooses the gateway, which can increase the number of hops and improve the reliability. When the link interrupts, $\mathrm{CH}$ UAV quickly finds a suitable route for information transmission because it maintains a routing list with the other CHs. Therefore, the EBMP has a lower average delay than MPBC and MPCR.

In Fig. 9, the average delay rises with the number of UAVs increase in one cluster at $v_{\max }=90 \mathrm{~m} / \mathrm{s}$. The possibility of congestion will be increased with the number of CM UAVs increase. For MPCR, the redundancy of information will produce in the cluster without ferry UAVs. When the number of CM UAVs is small, the average delay increased slowly. The average delay increases rapidly as the number of CM UAVs increases. MPBC has the same tendency with the EBMP when the number of CM UAVs is lower than 30 . After more than 30, the average delay rises rapidly. On the one hand, the CM UAVs contend slots with dynamic TDMA. On the other hand, $\mathrm{CH}$ UAV works as a receiver and transmitter for inter-communication, which makes enormous pressure on CH UAV. The contradiction frequently increases when the number of CM UAVs increases, which increases the packet loss probability of information for $\mathrm{CH}$ UAV, so that the average delay rises rapidly. The EBMP dynamically changes the gateway. On the one hand, it can alleviate the pressure of $\mathrm{CH} \mathrm{UAV}$. On the other hand, it can avoid a UAV serving as the gateway all the time. It can assign another gateway to choose a new path with light load when the path congested.

\section{Conclusion}

In this paper, we have proposed the EBMP algorithm for larger-scale UAV ad hoc networks and analyzed its performance. The proposed clustering algorithm uses power management and the Doppler shift to select CHs. Further we used the $\mathrm{CH}$ rotation strategy for a cluster to maintain the established clusters. Meanwhile, we designed a routing algorithm of the EBMP to optimize effectively the routing policy in intra-cluster and inter-cluster communication. The simulations show that the EBMP algorithm can do well in making the energy consumption in the whole network more balanced, and the structure of clusters trends stable. 
In the same simulation condition, the EBMP algorithm can achieve longer average $\mathrm{CH}$ lifetime compared with MPBC and MPCR algorithm. Besides, the numerical results show good performance of the EBMP algorithm in term of providing better throughput and lower average delay.

\section{References}

[1] Liu, M., Sun, Y.: Development Analysis of the military UAV technology abroad, Ship Electronic Engineering, 30(2010), 9, 22-27.

[2] Fan, W. T., Zhang, S., Ma, L. H., et al.: Energy balanced Multi hop aware Cooperative Geographic Routing for Wireless Ad Hoc Networks, Engineering Review, 36(2016), 2, 133-147.

[3] David, L.: Introduction to RF Stealth, Northwestern Polytechnic University Press, Xi'an, 2009.

[4] Wu, D., Zhu, F. X., Chen, X. C.: A Scheme of Real-time Multimedia Communication in ad hoc Networks Based on Probability Degree Clustering, Journal of Image and Graphics, 11(2006), 11, 1547-1551.

[5] Basu, P., Khan, N., Little, T.: A mobility based metric for clustering in mobile ad hoc networks, in Proc. Distrib. Comput. Syst, Mesa, 2001, 413-418.

[6] Zang, S. H.: Research of Clustering Algorithms and Cluster Based Routing Protocols for Ad
Hoc Networks (Master Dissertation), Information Engineering Nanchang Hangkong University, Nanchang, 2009.

[7] Ni, M., Zhong, Z. D., Zhao, D. M.: MPBC: A Mobility Prediction-Based Clustering Scheme for Ad Hoc Networks, IEEE Transactions on Vehicular Technology, 60(2011), 9, 4549-4559.

[8] Shu, J., Ge, Y. F., Liu, L. L., et al.: Mobility Prediction Clustering Routing in UAVs, International Conference on Computer Science and Network Technology, IEEE, 2011, 19831987

[9] Sergio, F., Guilherme, C., Filipe, F.: Assessing the capacity of man-portable UAVs for network access point localization, using RSSI link data, International Conference on Unmanned Aircraft Systems, Orlando, 2014, 355-364.

[10] Zheng, Y., Wang, Y. W., Li, Z. Z., et al.: A mobility and load aware OLSR routing protocol for UAV mobile ad-hoc networks, Proceedings of 15th IEEE Information and Communication Technologies Conference, Nanjing, 2014, 1-7.

[11] Chen, W. M., Zhou, X. Y.: A Clustering Algorithm for Mobile Ad Hoc Network, Chinese Journal of computers, 28(2005), 5, 864-869.

[12] Wei, R.: Research of Cluster-based Resource Allocation Scheme in High Dense Networks (Master Dissertation), Beijing University of Posts and Telecommunication, Beijing, 2015. 